\title{
Mechanisms of Acute Knee Injuries in Bouldering and Rock Climbing Athletes
}

\author{
Christoph Lutter, ${ }^{\star \dagger}$ MD, Thomas Tischer, ${ }^{\dagger} \mathrm{MD}, \mathrm{PhD}$, Carrie Cooper, ${ }^{\ddagger} \mathrm{DPT}$, Luisa Frank, ${ }^{\S} \mathrm{MD}$, \\ Thilo Hotfiel, ${ }^{\| \top}$ MD, PhD, Robert Lenz, ${ }^{\dagger}$ MD, and Volker Schöffl, ${ }^{\sharp \# * *+\dagger}$ MD, PhD \\ Investigation performed at Department of Orthopedic and Trauma Surgery, \\ Klinikum Bamberg, Bamberg, Germany
}

\begin{abstract}
Background: There is limited insight into the mechanisms of knee injuries in rock climbing and bouldering in noncompetitive and competitive athletes.
\end{abstract}

Purpose: To examine the traumatic mechanisms of injury, demographics, distribution, and severity of knee injuries in affected athletes.

Study Design: Case series; Level of evidence, 4.

Methods: During a 4-year period, we performed a retrospective multicenter analysis of acute knee injuries in competitive and noncompetitive climbing athletes. Traumatic mechanisms were inquired and severity levels, therapies, and outcomes recorded with visual analog scale, Tegner, Lysholm, and climbing-specific outcome scores.

Results: Within the observation period, 71 patients (35\% competitive athletes, 65\% noncompetitive athletes) with 77 independent acute knee injuries were recorded. Four trauma mechanisms were identified: high step (20.8\%), drop knee (16.9\%), heel hook (40.3\%), and (ground) fall (22.1\%). The leading structural damage was a medial meniscal tear (28.6\%), found significantly more often in the noncompetitive group. A specific climbing injury is iliotibial band strain during the heel hook position. Most injuries resulted from indoor bouldering (46.8\%). Surgical procedures were predominantly necessary in noncompetitive climbers. One year after the injury, the Tegner score was $5.9 \pm 0.8$ (mean \pm SD; range, 3-7); the Lysholm score was $97 \pm 4.8$ (range, 74-100); and the climbing-specific outcome score was $4.8 \pm 0.6$ (range, 2-5).

Conclusion: Increased attention should be placed on the climber's knee, especially given the worldwide rise of indoor bouldering. Sport-specific awareness and training programs for noncompetitive and competitive climbing athletes to reduce knee injuries should be developed, and sports medical supervision is mandatory.

Keywords: meniscal tear; anterior cruciate ligament (ACL) injury; injury mechanism; valgus; heel hook; sports injury

*Address correspondence to Christoph Lutter, MD, Orthopädische Universitätsklinik Rostock, Doberanerstraße 142, Rostock, 18057, Germany (email: christoph.lutter@googlemail.com).

${ }^{\dagger}$ Department of Orthopedics, University Medical Center, Rostock, Germany.

${ }^{\ddagger}$ Rev Physical Therapy, Salt Lake City, Utah, USA.

${ }^{\S}$ Department of Trauma Surgery, Friedrich Alexander University Erlangen-Nuremberg, Erlangen, Germany.

"Department of Orthopedics, Trauma and Hand Surgery, Klinikum Osnabrück, Osnabrück, Germany.

IDepartment of Orthopedic Surgery, Friedrich Alexander University Erlangen-Nuremberg, Erlangen, Germany.

"Section Sports Orthopedics \& Sports Medicine, Department of Orthopedic and Trauma Surgery, Klinikum Bamberg, Bamberg, Germany

**Wilderness Medicine Section, Department of Emergency Medicine, University of Colorado School of Medicine, Denver, Colorado, USA.

${ }^{+\dagger}$ School of Clinical and Applied Sciences, Leeds Beckett University, Leeds, UK.

Submitted June 22, 2019; accepted November 18, 2019.

The authors declared that they have no conflicts of interest in the authorship and publication of this contribution. AOSSM checks author disclosures against the Open Payments Database (OPD). AOSSM has not conducted an independent investigation on the OPD and disclaims any liability or responsibility relating thereto.

The American Journal of Sports Medicine

2020;48(3):730-738

DOI: $10.1177 / 0363546519899931$

(C) 2020 The Author(s)
With the worldwide growing interest and enthusiasm for rope climbing and bouldering, the sport has recently been selected as a new discipline for the upcoming 2020 Summer Olympics in Tokyo, Japan. Previous sports medicine research in rock climbing was dominated by studies focusing on sport-specific injury patterns of the upper extremity, such as finger pulley injuries or lumbrical muscle injuries, ${ }^{12,16}$ and injury incidences have been described. ${ }^{7,30}$ Overstrain injuries in climbing have been indicated to mainly affect the upper extremity, whereas acute traumatic injuries, such as ankle joint distortions, are predominantly seen on the lower extremities. ${ }^{5,16,24} \mathrm{Nev}$ ertheless, available literature emphasizes the fact that, overall, most injuries are of a minor grade. ${ }^{23}$ With 0.2 injuries per 1000 hours in recreational athletes ${ }^{25}$ and 3.1 in competitive climbing, ${ }^{26}$ incidence rates are documented to be low.

Rock climbing consists of 2 major subdisciplines that differ slightly in terms of physical demands and protection: "rope climbing" (ie, sport climbing) and "bouldering." While rope climbing requires a range of physical and psychological capacities, bouldering features a few very difficult movements at lower heights, thus demanding 
physical strength and technical skills. Both disciplines are performed on real rock and artificial walls. The main devices used in the protection of a fall are a rope and harness in sport climbing and bouldering pads for bouldering. Given the worldwide indoor bouldering boom and the changes in the athletes' preferences toward this subtype of rock climbing, we are currently finding an increase of absolute injury numbers, which are of a greater variety than those previously explored. ${ }^{2,5,11,19}$ Among these, knee injuries were recently reported to increase in numbers and relevance, and Josephsen et $\mathrm{al}^{8}$ reported a higher percentage of knee injuries in indoor bouldering as compared with outdoor bouldering. ${ }^{2,5,8,19,20}$ This change is explained by modern climbing and training methods causing a wider spectrum of climbing-related injuries. ${ }^{5,11,19,21}$ While knee injuries are among the most frequently diagnosed sports injuries in general, rock climbing and bouldering have not been identified as highly hazardous activities for these types of injuries. ${ }^{1,11,24}$ In a recent report from one of the world's biggest societies of sports medicine (GermanAustrian-Swiss Society for Orthopaedic Traumatologic Sports Medicine), the incidence of knee injuries from our database has doubled as compared with findings from 1998 to $2001{ }^{16,20}$ While $6.7 \%$ of all climbing injuries treated in our climbing-focused outpatient clinic in the recent past were knee injuries, other current studies report that up to $10 \%$ of total injuries are knee injuries among climbers. ${ }^{2}$ This finding strengthens the theory that there is an increase of knee injuries caused by rock climbing and bouldering. To date, traumatic mechanisms and demographics of knee injuries in bouldering and rope climbing are undefined. In contrast, detailed descriptions of traumatic mechanisms have been described in other sports, ${ }^{3,4,9}$ which have formed the basis for exercise-based injury prevention. ${ }^{13,14}$

The objective of this study was to analyze and describe various traumatic mechanisms, demographics, distribution, and severity of knee injuries in noncompetitive and competitive athletes. We hypothesized that there are various sport-specific mechanisms of knee injuries in rope climbing and bouldering.

\section{METHODS}

\section{Patients}

Between 2015 and 2018, we investigated all athletes (noncompetitive and competitive) with acute knee injuries related to rope climbing or bouldering (indoor and outdoor) treated in 2 specialized outpatient sports medicine clinics that are referral centers for climbing-related injuries (eg, German Alpine Club). Knee injuries caused by rope climbing or bouldering activities were defined as medical conditions forcing the athlete to rest from the sport because of pain or dysfunction and the necessity to seek help from a specialist. The study was approved by the institutional review board, and all patients provided informed consent.

\section{Diagnostic Algorithm}

Diagnoses were based on clinical investigation and radiological findings. All final diagnoses were reviewed and confirmed by the senior author (V.S.), who is a boardcertified orthopaedic surgeon and certified knee surgeon with $>25$ years of experience. Patients who were initially seen and treated in emergency departments were later reexamined in the outpatient sports medicine clinics. While one clinic is a trauma center located in one of Germany's biggest and most popular outdoor climbing areas, the second clinic is located between world-renowned climbing destinations in Utah. A standard questionnaire and examination protocol was conducted. Only patients experiencing pain during or after rope climbing or bouldering were included in the study. Tegner, Lysholm, and climbing-specific scores were used for evaluation of preinjury status of the affected knee (Tables 1 and 2).

\section{Classification}

The Union Internationale des Associations d'Alpinisme (UIAA) metric scale was used for evaluation of climbing levels, and the $\mathrm{V}$ scale was used for evaluation of bouldering levels as previously published. ${ }^{22,24}$ For classification of injury severities, the UIAA injury score was used following the UIAA MedCom recommendation. ${ }^{22}$

\section{Traumatic Mechanisms of Injury}

Athletes were asked to describe the exact mechanism of injury (MOI) that caused the pain, based on the body position and activity in the given climbing route and observations of their climbing partners. Subgroup analyses were then made per the different traumatic mechanisms (Figure 1).

\section{Therapeutic Algorithm}

Treatment consisted of an individualized posttraumatic injury therapy protocol for each athlete with gradual return to sports. In case of surgical treatment (eg, anterior cruciate ligament $[\mathrm{ACL}]$ reconstruction or meniscal repair), individual postoperative physical therapy protocols for each patient were used according to diagnostic and intraoperative findings. Physical therapy goals included range of motion, muscle strengthening, and dynamic stability exercises progressing to functional performance.

\section{Outcome}

All patients were seen for follow-up evaluations 6 and 12 weeks after the initial consultation and contacted after 1 year. The visual analog scale scores were collected at the initial visit and after 1 year. Tegner, Lysholm, and climbing-specific scores were recorded at 1 year. ${ }^{18}$ All patients self-reported preinjury conditions (Table 2).

\section{Statistical Analysis}

Microsoft Excel was used for data collection; statistical analyses were performed with SigmaStat software (Systat Software Inc, Version 11.0). Values were checked for 
TABLE 1

Patient Demographics, Treatment, and Outcome Grouped by Traumatic Mechanisms of Injury ${ }^{a}$

\begin{tabular}{|c|c|c|c|c|c|c|}
\hline & All Patients & High Step & Drop Knee & Heel Hook & Fall & $P$ Value \\
\hline Patients & 71 & 16 & 13 & 26 & 16 & \\
\hline Injuries & 77 & $16(20.8)$ & $13(16.9)$ & $31(40.3)$ & $17(22.1)$ & \\
\hline Age, y & $32.3 \pm 10(15-61)$ & $35.7 \pm 11.1(21-53)$ & $32.9 \pm 12.1(16-61)$ & $32.2 \pm 8.6(16-49)$ & $29.4 \pm 8.4(15-46)$ & NS \\
\hline Sex, men:women & $48: 29$ & $11: 5$ & $9: 4$ & $21: 10$ & $7: 10$ & \\
\hline Height, $\mathrm{cm}$ & $174.8 \pm 8.7(154-194)$ & $176.2 \pm 6.3(164-184)$ & $175.1 \pm 6.4(167-185)$ & $175.1 \pm 10.9(154-190)$ & $172.4 \pm 8.4(160-194)$ & NS \\
\hline Weight, kg & $66.7 \pm 11.9(39-91)$ & $68.4 \pm 11.3(51-86)$ & $70 \pm 13.6(52-91)$ & $68.1 \pm 11.4(49-90)$ & $59.6 \pm 11.1(39-82)$ & NS \\
\hline \multicolumn{7}{|l|}{ Level } \\
\hline Rope climbing $^{b}$ & $8.7 \pm 1.9(5-12)$ & $8.6 \pm 2(5-11.7)$ & $8.6 \pm 1.6(6-11)$ & $9 \pm 1.9(5-12)$ & $8.2 \pm 2.1(5-11.7)$ & NS \\
\hline Bouldering $^{c}$ & $7.6 \pm 4.3(0-15)$ & $7 \pm 4.6(0-15)$ & $6.8 \pm 3.6(2-12)$ & $8.6 \pm 4.5(0-15)$ & $6.9 \pm 4.6(1-14)$ & .03 \\
\hline \multicolumn{7}{|l|}{ Years } \\
\hline Rope climbing & $11 \pm 7.6(0-31)$ & $11.4 \pm 8.8(0-30)$ & $10.9 \pm 8.6(1-31)$ & $12.2 \pm 6.7(2-24)$ & $8.5 \pm 7.1(1-20)$ & NS \\
\hline Bouldering & $9.2 \pm 7.3(0-30)$ & $11.2 \pm 8.9(0-30)$ & $5.3 \pm 4.8(0-15)$ & $10.7 \pm 7(0-20)$ & $7.6 \pm 6.9(0-20)$ & .03 \\
\hline Bouldering $^{d}$ & $58.2 \pm 31.1(0-100)$ & $51.2 \pm 29.0(0-100)$ & $41.9 \pm 28.7(0-90)$ & $62.6 \pm 31.7(0-100)$ & $69.1 \pm 29.6(0-100)$ & .045 \\
\hline \multicolumn{7}{|l|}{ Competitive level, yes:no } \\
\hline Competing & $28: 49(36.4)$ & $5: 11(6.5)$ & $3: 10(3.8)$ & $14: 17(18.2)$ & $6: 11(7.8)$ & \\
\hline World Cup & $17: 60(22)$ & $3: 13(3.9)$ & $1: 12(1.3)$ & $9: 22(11.7)$ & $4: 13(5.2)$ & \\
\hline Training, $\mathrm{h} / \mathrm{wk}$ & $12.9 \pm 10.7(0-40)$ & $11.3 \pm 10.7(0-30)$ & $11.2 \pm 8.9(3-35)$ & $15.1 \pm 12.4(2-40)$ & $11.8 \pm 8.7(2-30)$ & $<.001$ \\
\hline Warm-up routine, yes:no & $61: 16$ & $11: 5$ & $11: 2$ & $26: 5$ & $13: 4$ & NS \\
\hline Alternative sports yes:no & $54: 23$ & $12: 4$ & $10: 3$ & $21: 10$ & $11: 6$ & \\
\hline \multicolumn{7}{|l|}{ Rope climbing injury } \\
\hline Indoor & 4 & 1 & 2 & 1 & 0 & \\
\hline Outdoor & 20 & 10 & 5 & 3 & 2 & \\
\hline \multicolumn{7}{|l|}{ Bouldering injury } \\
\hline Indoor & 36 & 5 & 3 & 17 & 11 & \\
\hline Outdoor & 17 & 0 & 3 & 10 & 4 & \\
\hline UIAA injury score & $2.1 \pm 0.4(1-3)$ & $1.9 \pm 0.3(1-2)$ & $1.9 \pm 0.3(1-2)$ & $2 \pm 0(2-2)$ & $2.4 \pm 0.5(2-3)$ & $<.001$ \\
\hline Affected side, right:left & $33: 44$ & $7: 9$ & $6: 7$ & $12: 19$ & $8: 9$ & \\
\hline Surgical treatment, yes:no & $26: 51$ & $6: 10$ & $4: 9$ & $5: 26$ & $11: 6$ & \\
\hline \multicolumn{7}{|l|}{ Score $^{e}$} \\
\hline VAS & $1 \pm 0.7(0-3)$ & $0.7 \pm 0.7(0-2)$ & $0.2 \pm 0.4(0-1)$ & $0.4 \pm 0.7(0-3)$ & $0.8 \pm 0.8(0-2)$ & $\mathrm{NS}$ \\
\hline Tegner & $5.9 \pm 0.8(3-7)$ & $6 \pm 0.8(5-7)$ & $5.9 \pm 0.5(5-7)$ & $6 \pm 0.9(3-7)$ & $5.8 \pm 0.9(4-7)$ & NS \\
\hline Lysholm & $97 \pm 4.8(74-100)$ & $97.9 \pm 3(91-100)$ & $97.9 \pm 3.6(90-100)$ & $97.3 \pm 4.9(79-100)$ & $95.3 \pm 6.4(74-100)$ & NS \\
\hline Climbing & $4.8 \pm 0.6(2-5)$ & $4.8 \pm 0.4(4-5)$ & $4.9 \pm 0.3(4-5)$ & $4.9 \pm 0.6(2-5)$ & $4.5 \pm 0.8(2-5)$ & NS \\
\hline
\end{tabular}

${ }^{a}$ Data are provided as $\mathrm{n}(\%)$ or mean $\pm \mathrm{SD}$ (range). NS, not significant; UIAA, Union Internationale des Associations d'Alpinisme; VAS, visual analog scale for pain.

${ }^{b}$ Rope climbing levels graded according to UIAA metric scale.

${ }^{c}$ Bouldering levels graded according to $\mathrm{V}$ scale.

${ }^{d}$ Percentage of bouldering in climbing/training routine.

${ }^{e}$ One year after injury.

normality with the Shapiro-Wilk test. To determine the difference among groups, a $t$ test or rank sum test was used depending on normal distribution. A nonparametric Kruskal-Wallis 1-way analysis of variance on ranks was used for nonnormally distributed data among several groups. Unless stated otherwise, data are expressed as mean and standard deviation with range, as appropriate. Subgroup analyses were made for 4 trauma mechanisms as described before. $P$ values $<.05$ were considered as statistically significant.

\section{RESULTS}

During the 4-year period, we treated 71 patients (45 [63.4\%] male, 26 [36.6\%] female) with 77 independent knee injuries caused by rope climbing or bouldering (Table 1). Twenty-five (35.2\%) of the athletes performed the sport on a competitive level; among those, $15(21.1 \%)$ were World Cup-level athletes. Only 5 injuries occurred during a competition (1 during national competition, 4 during World
Cup competition), while 66 injuries occurred during training. Rope climbing and bouldering skill levels as well as demographic information are shown in Table 1. The mean UIAA injury score was $2.1 \pm 0.4$ (range, $1-3$ ), and among the 7 injured athletes with an UIAA score of 3 , two were male. Patients with a UIAA score of 3 experienced ACL ruptures with meniscal and collateral ligament tears or joint fractures as a result of a fall. None of the athletes had a UIAA grade 4 or 5 injury, and none of them died (UIAA 6). Rope climbing level (UIAA level) and climbing experience (climbing years) did not vary significantly between men and women, but men had a significantly higher bouldering level (V scale, $P=.03$ ) and experience (bouldering years, $P=.03$ ) than women.

\section{Traumatic MOls}

Four types of traumatic MOIs were identified: the high step position (Figure 1A), the drop knee position (Figure 1B), the heel hook position (Figure 1C), and a fall to the ground (Figure 1D). No other MOIs were reported. Apart 

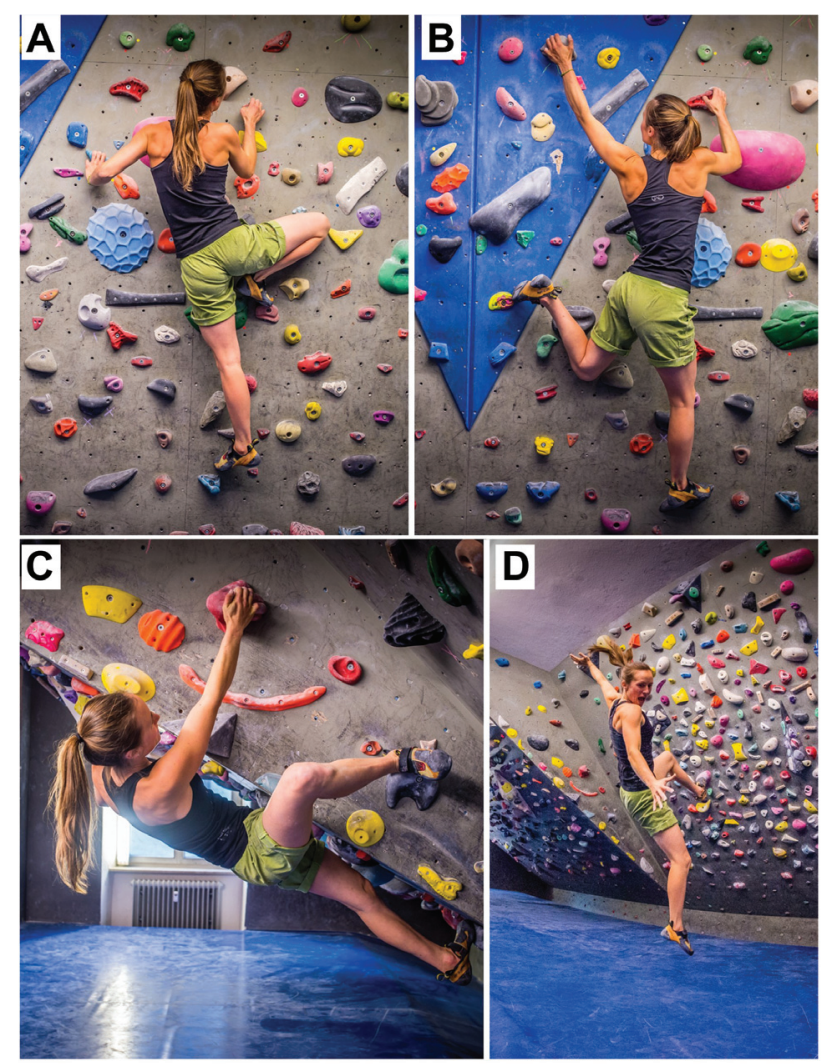

Figure 1. (A) High step position of the right leg/knee during bouldering. While the left leg is fully unloaded, the majority of the athlete's weight is on the right leg with a fully flexed knee. (B) Drop knee position of the left lower extremity leading to a high mechanical load on the menisci. (C) Heel hook position during bouldering (right knee). The heel is used to apply pressure onto the hold while pulling on the foot by flexing the hamstrings. In addition, the knee is rotated outwardly, applying a high load onto the knee. (D) A fall during bouldering can cause severe knee injuries, even from lower heights in cases of uncontrolled falling, insufficient ground protection, or insufficient body tension attributed to fatigue. from the fall, all body positions are commonly known techniques in climbing, although the only MOI previously described in the literature is the heel hook position. ${ }^{21}$

The high step position features a squat position of the affected leg and knee during rope climbing or bouldering. One leg is fully unloaded and mostly extended, and the majority of the athlete's weight is on the other leg, with a fully flexed knee and an externally rotated, flexed, and abducted hip (Figure 1A). An internally rotated hip with a flexed knee characterizes the drop knee position during rope climbing and bouldering. For improved body positioning, the athlete loads the extremity, which leads to a high mechanical load on the medial meniscus, especially during combined extension and rotation under load (Figure 1B). During the heel hook position, the heel is used to apply pressure onto the hold while pulling on the foot by flexing the knee via a strong hamstring contraction. In addition, the knee is rotated laterally, applying a high load to the $\mathrm{knee}^{21}$ (Figure 1C). A fall as a traumatic MOI for severe knee injuries results from uncontrolled falling (eg, load in combination with rotational movements), insufficient ground protection, or insufficient body control owing to fatigue (eg, reduced core stability or limited neuromuscular control causing valgus loading of the knee), even from lower heights (Figure 1D).

With $46.8 \%$ of all injuries, indoor bouldering was the main cause of injury, followed by outdoor rope climbing $(26 \%)$, outdoor bouldering $(22.1 \%)$, and indoor rope climbing $(5.2 \%)$ (Table 1$)$.

\section{Injury Distribution}

Injury distribution is presented in Table 3. The leading structural damage in the knee was a medial meniscal tear, which was the leading diagnosis in $28.6 \%(\mathrm{n}=22)$ of all injuries. These injuries were predominantly caused by the high step, drop knee, and heel hook positions. Iliotibial band sprains were present in $19.5 \%(n=15)$ of all injuries. This type of injury resulted exclusively from heel hook positions. ACL tears combined with medial collateral ligament and medial meniscal injuries were

TABLE 2

Sport-Specific Outcome Score After Climbing Injuries ${ }^{a}$

\begin{tabular}{|c|c|c|}
\hline Grade & Outcome & Posttraumatic Climbing Capacity \\
\hline $\mathrm{V}$ & Excellent & $\begin{array}{l}\text { Full load capacity of the former injured joint/limb after } 12 \mathrm{mo} \text {, no subjective strength deficit, regain of full climbing } \\
\text { ability/preinjury climbing level, no pain }\end{array}$ \\
\hline IV & Good & $\begin{array}{l}\text { Full load capacity of the former injured joint/limb after } 12 \mathrm{mo} \text {, subjectively minor strength deficit, regain of full } \\
\text { climbing ability/preinjury climbing level, minor pain }\end{array}$ \\
\hline III & Satisfactory & $\begin{array}{l}\text { Minor restricted load capacity of the former injured joint/limb, subjectively major strength deficit of the former } \\
\text { injured joint, regain of full climbing ability/preinjury UIAA climbing level minus } 1 \text { UIAA grade, minor pain }\end{array}$ \\
\hline II & Fair & $\begin{array}{l}\text { Major restricted load capacity of the former joint/limb, strength deficit and restricted ability to use the former } \\
\text { injured joint while climbing, major decrease in climbing ability and grade, frequent pain }\end{array}$ \\
\hline I & Poor & Climbing not possible anymore \\
\hline
\end{tabular}

${ }^{a}$ Modified from Schöffl et al. ${ }^{23}$ UIAA, Union Internationale des Associations d'Alpinisme. 
TABLE 3

Injury Distribution Listed by Main Diagnosis ${ }^{a}$

\begin{tabular}{|c|c|c|c|c|c|}
\hline Injury Type & \multicolumn{5}{|c|}{ Mechanism of Injury, n (\%) } \\
\hline Iliotibial band sprain $^{b}$ & $15(19.5)$ & & & $15(48.4)$ & \\
\hline ACL tear ${ }^{c}$ & $7(9.1)$ & & & & $7(41.2)$ \\
\hline LCL tear & $6(7.8)$ & & $1(7.7)$ & $5(16.1)$ & \\
\hline Lateral meniscal tear & $4(5.2)$ & $1(6.3)$ & $2(15.4)$ & $1(3.2)$ & \\
\hline Cartilage injury & $3(3.9)$ & $2(12.5)$ & & $1(3.2)$ & \\
\hline Others & $3(3.9)$ & $1(6.3)$ & & & $2(11.8)$ \\
\hline \multicolumn{6}{|l|}{$\mathrm{ACL}$} \\
\hline Rupture & $2(2.6)$ & & & & $2(11.8)$ \\
\hline Partial tear & $2(2.6)$ & & & $1(3.2)$ & $1(5.9)$ \\
\hline
\end{tabular}

${ }^{a} \mathrm{ACL}$, anterior cruciate ligament; MCL, medial collateral ligament.

${ }^{b}$ Heel hook injury as described before. ${ }^{15}$

${ }^{c}$ ACL tear with medial meniscal tear \pm MCL tear.

detected in $9.1 \%(\mathrm{n}=7)$ and isolated ACL tears $(\mathrm{n}=2)$ or partial ACL tears $(n=2)$ in $2.6 \%$. Ninety-one percent of injuries that caused at least a partial tear of the ACL resulted from a fall to the ground. Lateral collateral ligament $(7.8 \% ; \mathrm{n}=6)$ or isolated lateral meniscal $(5.2 \% ; \mathrm{n}=$ 4) tears occurred less frequently. Distribution of all other injuries is shown in Table 3.

\section{Outcomes}

All athletes had returned to sports within 12 months. One year after the injury, the mean visual analog scale for pain score was $1 \pm 0.7$ (range, $0-3$ ); the Tegner Score, $5.9 \pm 0.8$ (range, 3-7); the Lysholm score, $97 \pm 4.8$ (range, 74-100); and the climbing-specific outcome score, $4.8 \pm 0.6$ (range, $2-5$ ). The mean decrease in the Tegner score was 0.2 , while the mean Lysholm score decreased by 2.8 . The injuryrelated mean drop in the climbing-specific outcome score was 0.2 . Two female athletes experienced a prolonged healing period with a major decrease in climbing ability and grade as well as ongoing pain 1 year after meniscal repair $(n=1)$ and open reduction and internal fixation $(n=1)$. Outcome scores separated by trauma mechanism are shown in Table 1.

\section{Between-Group Comparison of Traumatic MOls}

High step and drop knee injuries were more common during rope climbing, whereas heel hook and fall injuries were more often caused by bouldering. Bouldering ability levels varied significantly among groups $(P=.03)$. Patients who were injured during a heel hook had the highest ability level, and patients injured during a fall had the lowest bouldering ability level. Experience in bouldering (years) varied significantly, being highest in the high step group and lowest in the drop knee group $(P=.03)$. Patients who were injured during a heel hook had the highest training volume per week (15.1 hours), while athletes from the drop knee group had the lowest (11.2 hours). While no significant difference was found in rope climbing experience (years) among the groups, the athletes' experience in bouldering varied significantly. The longest experience in bouldering was in the high step group and the lowest in the drop knee group. The percentage of bouldering among the athletes' entire climbing practice was also significantly different among the groups $(P=.45)$ : while athletes injured during heel hook or fall were predominantly bouldering athletes, patients injured in high step or drop knee reported spending less time with bouldering. Athletes who got injured in a fall or heel hook thereby reported spending almost $70 \%$ and $63 \%$ of their climbing time in bouldering, respectively. Athletes with a knee injury from a drop knee or high step reported spending only $42 \%$ and $51 \%$ of their climbing time in bouldering, respectively. The UIAA injury score was significantly higher in the fall group (UIAA score, 2.4) as compared with the other groups.

\section{Noncompetitive and Competitive Athletes}

Competitive athletes $(35 \%)$ were significantly younger and lighter than the noncompetitive athletes. Rope climbing and bouldering levels, experience, training hours, and warm-up routines were all significantly higher in the 
TABLE 4

Comparison of Knee Injuries in Noncompetitive and Competitive Athletes ${ }^{a}$

\begin{tabular}{|c|c|c|c|}
\hline & Noncompetitive & Competitive & $P$ Value \\
\hline Patients & 46 & 25 & \\
\hline Injuries & 49 & 28 & \\
\hline Age, y & $35.8 \pm 9.9(16-61)$ & $26.5 \pm 6.3(15-39)$ & $<.001$ \\
\hline Sex, men:women & $29: 17$ & $16: 9$ & \\
\hline Height, cm & $176 \pm 9.2(154-194)$ & $173 \pm 7.6(160-186)$ & NS \\
\hline Weight, kg & $70.2 \pm 11.5(49-91)$ & $61.1 \pm 10.4(39-79)$ & .006 \\
\hline \multicolumn{4}{|l|}{ Level } \\
\hline Rope climbing ${ }^{b}$ & $7.7 \pm 1.6(5-10.3)$ & $10.5 \pm 0.7(9-12)$ & $<.001$ \\
\hline Bouldering $^{c}$ & $5.1 \pm 3.2(0-11)$ & $11.9 \pm 2.3(6-15)$ & $<.001$ \\
\hline \multicolumn{4}{|l|}{ Years } \\
\hline Rope climbing & $9.2 \pm 8.1(0-31)$ & $14.2 \pm 5.3(4-24)$ & .001 \\
\hline Bouldering & $6.5 \pm 7.1(0-30)$ & $14 \pm 5(4-20)$ & $<.001$ \\
\hline Bouldering $^{d}$ & $54 \pm 36(0-100)$ & $65.4 \pm 18.4(30-100)$ & NS \\
\hline Training, h/wk & $7.2 \pm 5.2(0-20)$ & $22.9 \pm 10.6(6-40)$ & $<.001$ \\
\hline Warm-up routine, yes:no & $32: 14$ & $24: 1$ & .009 \\
\hline Alternative sports, yes:no & $29: 17$ & $21: 4$ & NS \\
\hline \multicolumn{4}{|l|}{ Rope climbing injury } \\
\hline Indoor & 2 & 2 & NS \\
\hline Outdoor & 17 & 3 & NS \\
\hline \multicolumn{4}{|l|}{ Bouldering injury } \\
\hline Indoor & 21 & 15 & NS \\
\hline Outdoor & 9 & 8 & NS \\
\hline \multicolumn{4}{|l|}{ Trauma mechanism } \\
\hline High step & 11 & 5 & NS \\
\hline Drop knee & 10 & 3 & NS \\
\hline Heel hook & 17 & 14 & NS \\
\hline Fall & 11 & 6 & NS \\
\hline \multicolumn{4}{|l|}{ Injury type } \\
\hline Medial meniscal tear & 22 & 2 & .001 \\
\hline Iliotibial band sprain & 7 & 8 & NS \\
\hline ACL tear & 5 & 6 & NS \\
\hline LCL tear & 2 & 4 & NS \\
\hline Other & 13 & 8 & \\
\hline UIAA injury score & $2.1 \pm 0.3(1-3)$ & $2 \pm 0.4(1-3)$ & NS \\
\hline Affected side, right:left & $20: 29$ & $13: 15$ & NS \\
\hline Surgical treatment, yes:no & $21: 28$ & $5: 23$ & .047 \\
\hline \multicolumn{4}{|l|}{ Score ${ }^{e}$} \\
\hline VAS & $0.6 \pm 0.8(0-3)$ & $0.3 \pm 0.5(0-1)$ & NS \\
\hline Tegner & $5.6 \pm 0.6(3-6)$ & $6.6 \pm 0.6(5-7)$ & $<.001$ \\
\hline Lysholm & $95.9 \pm 5.4(74-100)$ & $99.4 \pm 1.5(95-100)$ & $<.001$ \\
\hline Climbing & $4.7 \pm 0.7(2-5)$ & $4.9 \pm 0.3(4-5)$ & NS \\
\hline
\end{tabular}

${ }^{a}$ Data are provided as No. or mean \pm SD (range). ACL, anterior cruciate ligament; LCL, lateral collateral ligament; NS, not significant; UIAA, Union Internationale des Associations d'Alpinisme; VAS, visual analog scale for pain.

${ }^{b}$ Rope climbing levels graded according to UIAA metric scale.

${ }^{c}$ Bouldering levels graded according to $\mathrm{V}$ scale.

${ }^{d}$ Percentage of bouldering in climbing/training routine.

${ }^{e}$ One year after injury.

competitive group of athletes as compared with the noncompetitive athletes. No significant difference in traumatic MOI was found between groups, although medial meniscal tears were significantly more frequent in noncompetitive athletes. Surgical procedures were required significantly more often in the noncompetitive group as compared with the competitive group. Tegner and Lysholm scores were significantly better in competitive climbers after 1 year (Table 4).

\section{DISCUSSION}

This is the first study to describe traumatic MOIs, injury patterns, and outcome measurements of acute knee injuries in noncompetitive and competitive rock climbers.

Four types of MOI were detected: the high step position, the drop knee position, the heel hook technique, and uncontrolled landing during a fall. In accordance with the Josephsen et $\mathrm{al}^{8}$ study, the majority of knee injuries 
in our study (47\%) happened while indoor bouldering, even though both study centers are located in regions known for their famous outdoor rope climbing ${ }^{8}$ (see Table 1). In total, injuries caused by bouldering activities represented $69 \%$ of all knee injuries within our study, which strengthens the theory that bouldering seems to be more prone to causing knee injuries than climbing with a rope. The reason for this might be that bouldering routes normally consist of few but very hard moves, which require strong body tension, difficult body positioning, and strength, especially placing the meniscus under enormous stress. Furthermore, falls to the ground are common in bouldering. Climbing routes, however, are generally much longer and require other skills, such as endurance, while a rope protects the athlete from a fall to the ground. ${ }^{11}$

Studies from other sports, including handball and basketball, have shown that insufficient landing with immediate valgus loading is a contributing factor in the ACL injury mechanism and that various intrinsic factors predispose female athletes for this type of injury. ${ }^{9,10,29}$ In our study, knee injuries caused by a fall had a significantly higher UIAA score than injuries from other trauma mechanisms and were predominantly found in female athletes. Furthermore, those injuries were mainly found in athletes with less experience and potentially less body control and stability strength while landing ${ }^{9,19}$ (see Table 1). Indoor bouldering gyms are widely available, and unexperienced athletes tend to choose indoor bouldering because of the ease of access. ${ }^{19}$

As in other sports in which various injury patterns correlate with the underlying traumatic MOI (eg, downhill skiing), ${ }^{28}$ we found several climbing techniques to predominantly cause typical injuries. Iliotibial band sprains at the lateral condyle were caused exclusively by heel hook positions (Table 3, Figure 1C), which seems reasonable as the heel hook position is the only body position among the described mechanisms in which the iliotibial band is fully tightened and eventually glides over the lateral condyle of the femur under tension. ${ }^{21}$ All ACL tears within our study group happened in cases of uncontrolled falls onto the ground. The mean bouldering level in athletes of the fall group was lower than that in the other groups (Table 3; Figure 1, C and D). This is an interesting fact and goes with the current increase of more severe injuries in beginners. ${ }^{19}$ Nevertheless, we saw ACL tears also happen during World Cup events. ${ }^{2,5,19}$ The mean climbing and bouldering level (UIAA metric scale, 8.7; $\mathrm{V}$ scale, 7.6) within the overall patient population of this study was consistent with other studies from our group but might not fully represent the climbing community as a whole. ${ }^{16}$ Given the current wave of beginners in bouldering, even more injuries based on falls under insufficient body control might be expected. ${ }^{19}$

Medial meniscal tears were predominantly caused by the high step, the drop knee, and the heel hook positions (Table 3). While none of the athletes reported preexisting symptoms in the affected knee, asymptomatic preexisting meniscal degeneration with an acute aggravation caused by the specific motion needs to be considered. The athlete stresses the leg in a fully flexed knee in the drop knee and high step positions (see Figure 1, A and B), causing a high load on the meniscus. The heel hook technique rather causes a shear stress to the menisci by a slightly flexed but fully loaded knee under tension ${ }^{21}$ (see Figure 1C). That athletes experiencing knee injuries while performing a heel hook had a high climbing or bouldering level as well as a high weekly training workload might strengthen the theory that stronger climbers use this technique more aggressively than others and thereby injure the knee. ${ }^{21}$ For all 3 MOIs, a peak load is suspected to cause the injury. It can be speculated that it is the moment of maximum contraction of the knee flexor muscles that causes the injury in cases of heel hook-related injuries. We suspect injury-causing events for the drop knee and high step positions to be different. In both body positions, we can assume that the injury happens within the moment when the athlete releases one hand to reach the next hold, leading to an extra load on the lower extremities. A slipping foot from the contralateral foothold causing eccentric stress onto the other side could cause peak loads to the knee. Eccentric peak loads have also been found to cause finger injuries in rock climbers. ${ }^{15}$ Furthermore, insufficient technical skills and fatigue might cause harmful rotational motion of the knee in experienced athletes.

In a recent systematic review, Eberbach et $\mathrm{al}^{6}$ reported that $89 \%$ of patients achieved a return to sports at the preinjury level after meniscal repair and that the mean return occurred within 4.3 to 6.5 months (postoperative Tegner score, $6.2 \pm 0.8) .{ }^{6}$ While not all of our athletes with meniscal tears received a meniscal repair, the high sport-specific outcome score of 4.8 and the Tegner score of 5.9 go with the findings from Eberbach et al. Seijas et $\mathrm{al}^{27}$ recently analyzed return to preinjury Tegner levels after ACL reconstructions and reported a mean Tegner level of 5.9 at 12 months after surgery. All of our patients with ACL injuries had returned to sport after 1 year, and the mean Tegner score was 6.0. Exact determination of return-to-sport time points was not possible, as most of the athletes reported that they returned to climbing gradually during the rehabilitation process under awareness and care for the injured knee. In contrast to contact sports requiring running or jumping, climbing is a mostly predictable noncontact sport, which allows the athlete to perform it with reduced stress on the leg. Some athletes might even reach their preinjury climbing level while still gently using the affected knee in some movements. This might be the reason for a relatively quick return to sport for our athletes.

Between-group comparison of competitive and noncompetitive athletes revealed 2 interesting findings. First, noncompetitive athletes had significantly more medial meniscal tears and a slightly higher UIAA injury score. Hypothetically, this could be due to a lower level of body awareness and control in the noncompetitive group. Second, the noncompetitive group underwent more surgical procedures than the competitive group. The reasons remain unclear but might be influenced by the significantly lower age and body weight of the competitive group; as medial meniscal tears increase in prevalence with age, 
asymptomatic preexisting meniscal lesions should also be considered.

To prevent reinjury of the affected knee during the return-to-sport process, rope climbing should be preferred to bouldering to avoid falls and direct impact on the knee. In case of bouldering activities with a previously injured knee, "down climb" (cautious descent) or "top out" (alternative easy descent) should be preferred to jumping down on the mat after the successful ascent. Rope climbing on an overhanging wall has been described as having a lower injury risk for the lower extremities ${ }^{17}$ and should therefore be preferred to rope climbing on vertical walls. Falls on overhangs are stopped exclusively by the rope, whereas falls during rope climbing on a vertical wall can cause a swing into the wall. ${ }^{17}$

Based on the current findings, it may be possible to develop training programs for noncompetitive and competitive climbing athletes to address muscle weaknesses not trained exclusively through climbing. Most climbers neglect the leg muscles completely in their training routine. Active training of the knee stabilizers for improved joint control and stretching techniques (eg, iliotibial band) may be advisable. As an effect of this addition, excessive load on the knee during the described climbing techniques might be reduced. Improved psychomotor skills and body control might better manage and reduce the risk of injury during falls.

\section{Study Limitations}

This study has several limitations. The patient population was not fully homogeneous, as most of the athletes came from the local climbing areas but a few were from farther away. The kind of climbing performed in certain regions and the accessibility to climbing/bouldering gyms might influence the type of injuries. The main limitation might be the fact that there is a bias within the patient population: athletes who experience severe knee trauma most likely seek help at the closest medical facility, especially in cases of fractures or acute ligament injuries, rather than consulting a facility specialized on climbing injuries. The number of severe knee injuries, particularly in indoor bouldering, is expected to be even higher than presented in this study. Conclusions cannot be reached about the incidence of the injuries among the climbing and bouldering population at large, and the injury distribution may not be generalizable to other populations. Injury mechanisms were based on athletes' descriptions and could not be independently verified. However, this is the first study that highlights the knee injuries resulting directly from rock climbing and bouldering.

\section{CONCLUSION}

We identified 4 distinctive traumatic MOIs of knee injuries, referred to as the high step, drop knee, heel hook, and (uncontrolled) fall. Meniscal tears, iliotibial band sprains, and ACL injuries were the leading diagnoses, and the return to sport was quick overall. Noncompetitive athletes had significantly more medial meniscal tears than competitive athletes and underwent more surgical procedures.

Increased attention should be placed on the climber's knee, especially given the worldwide rise of indoor bouldering. Sport-specific awareness training programs for noncompetitive and competitive climbing athletes to avoid excessive load on the knee should be developed, and sports medical supervision is mandatory.

\section{ACKNOWLEDGMENT}

The authors thank Victoria Lenz and Enrico Haase for photographs.

\section{REFERENCES}

1. Arendt E, Dick R. Knee injury patterns among men and women in collegiate basketball and soccer: NCAA data and review of literature. Am J Sports Med. 1995;23(6):694-701.

2. Asakawa D, Sakamoto M. Retrospective survey of sport climbing injuries and self-care in the Gunma prefecture. J Phys Ther Sci. 2019;31:332-335.

3. Bakken A, Bere T, Bahr R, Kristianslund E, Nordsletten L. Mechanisms of injuries in World Cup Snowboard Cross: a systematic video analysis of 19 cases. Br J Sports Med. 2011;45(16):1315-1322.

4. Bere T, Florenes TW, Krosshaug T, et al. Mechanisms of anterior cruciate ligament injury in World Cup alpine skiing: a systematic video analysis of 20 cases. Am J Sports Med. 2011;39(7):1421-1429.

5. Buzzacott P, Schöffl I, Chimiak J, Schöffl V. Rock climbing injuries treated in US emergency departments, 2008-2016. Wilderness Environ Med. 2019;30(2):121-128.

6. Eberbach H, Zwingmann J, Hohloch L, et al. Sport-specific outcomes after isolated meniscal repair: a systematic review. Knee Surg Sports Traumatol Arthrosc. 2018;26(3):762-771.

7. Jones G, Asghar A, Llewellyn DJ. The epidemiology of rock-climbing injuries. Br J Sports Med. 2008;42(9):773-778.

8. Josephsen G, Shinneman S, Tamayo-Sarver J, et al. Injuries in bouldering: a prospective study. Wilderness Environ Med. 2007;18:271-280.

9. Koga H, Nakamae A, Shima $Y$, et al. Mechanisms for noncontact anterior cruciate ligament injuries: knee joint kinematics in 10 injury situations from female team handball and basketball. Am J Sports Med. 2010;38(11):2218-2225.

10. Kramer KL, Jani L, Grifka J, Scharf HP, Schleberger R, Zacher J. Guidelines in orthopaedic surgery: a first step. Orthopade. 1999; 28(3):236-242.

11. Lutter C, El-Sheikh Y, Schöffl I, Schöffl V. Sport climbing: medical considerations for this new Olympic discipline. $\mathrm{Br} J$ Sports Med. 2017;51(1):2-3.

12. Lutter C, Schweizer A, Schöffl V, Romer F, Bayer T. Lumbrical muscle tear: clinical presentation, imaging findings and outcome. J Hand Surg Eur Vol. 2018;43(7):767-775.

13. Myklebust G, Engebretsen L, Braekken IH, Skjolberg A, Olsen OE, Bahr R. Prevention of noncontact anterior cruciate ligament injuries in elite and adolescent female team handball athletes. Instr Course Lect. 2007;56:407-418.

14. Olsen OE, Myklebust G, Engebretsen L, Holme I, Bahr R. Exercises to prevent lower limb injuries in youth sports: cluster randomised controlled trial. Br Med J. 2005;330(7489):449.

15. Schöffl I, Oppelt K, Jungert J, et al. The influence of concentric and eccentric loading on the finger pulley system. J Biomech. 2009; 42(13):2124-2128. 
16. Schöffl V, Hochholzer T, Winkelmann HP, Strecker W. Pulley injuries in rock climbers. Wilderness Environ Med. 2003;14(2):94-100.

17. Schöffl V, Kupper T. Feet injuries in rock climbers. World J Orthop. 2013;4(4):218-228.

18. Schöffl V, Kupper T, Hartmann J, Schöffl I. Surgical repair of multiple pulley injuries-evaluation of a new combined pulley repair. $J$ Hand Surg Am. 2012;37(2):224-230.

19. Schöffl V, Lutter C. The "newbie" syndrome. Wilderness Environ Med. 2017;28(4):377-380.

20. Schöffl V, Lutter C. Sportklettern auf dem Weg zu Olympia. https:// www.gots.org/blog/2019/04/08/sportklettern-auf-dem-weg-zu-olympia/. Accessed April 22, 2019.

21. Schöffl V, Lutter C, Popp D. The "heel hook"-a climbing-specific technique to injure the leg. Wilderness Environ Med. 2016;27(2): 294-301.

22. Schöffl V, Morrison A, Hefti U, Ullrich S, Kupper T. The UIAA medical commission injury classification for mountaineering and climbing sports. Wilderness Environ Med. 2011;22(1):46-51.

23. Schöffl V, Morrison A, Schöffl I, Kupper T. The epidemiology of injury in mountaineering, rock and ice climbing. Med Sport Sci. 2012;58:17-43.
24. Schöffl V, Popp D, Kupper T, Schöffl I. Injury trends in rock climbers: evaluation of a case series of 911 injuries between 2009 and 2012. Wilderness Environ Med. 2015;26(1):62-67.

25. Schöffl VR, Hoffmann G, Kupper T. Acute injury risk and severity in indoor climbing-a prospective analysis of 515,337 indoor climbing wall visits in 5 years. Wilderness Environ Med. 2013;24(3):187-194.

26. Schöffl VR, Kuepper T. Injuries at the 2005 world championships in rock climbing. Wilderness Environ Med. 2006;17(3):187-190.

27. Seijas R, Ares O, Sallent A, Alvarez P, Cusco X, Cugat R. Return to prelesional Tegner level after anatomic anterior cruciate ligament reconstruction. Arch Orthop Trauma Surg. 2016;136(12):1695-1699.

28. Shea KG, Archibald-Seiffer N, Murdock E, et al. Knee injuries in downhill skiers: a 6-year survey study. Orthop J Sports Med. 2014;2(1):2325967113519741.

29. Sutton KM, Bullock JM. Anterior cruciate ligament rupture: differences between males and females. J Am Acad Orthop Surg. 2013; 21(1):41-50.

30. Woollings KY, McKay CD, Emery CA. Risk factors for injury in sport climbing and bouldering: a systematic review of the literature. $\mathrm{Br} \mathrm{J}$ Sports Med. 2015;49(17):1094-1099.

For reprints and permission queries, please visit SAGE's Web site at http://www.sagepub.com/journalsPermissions.nav. 